\title{
Eine personenzentrierte Betrachtung der Entwicklung des Fachinteresses Deutsch, Mathematik und Englisch von Jahrgangsstufe 4 bis 11
}

\author{
Irene M. Schiefer ${ }^{1}$, Sarah Becker ${ }^{1}$, Cordula Artelt ${ }^{2}$ \\ Universität Bamberg \\ 2 Leibniz-Institut für Bildungsverläufe, Bamberg
}

\begin{abstract}
Zusammenfassung: Forschungsarbeiten zur Interessensentwicklung konnten vielfach zeigen, dass Schülerinnen und Schüler mit undifferenziert hohen schulfachbezogenen Interessen in die Schule eintreten, die mittleren Interessen im weiteren Schulverlauf jedoch stetig absinken. Neuere Studien verweisen auf einen stagnierenden Interessensabfall ab Jahrgangsstufe 7 und einen leichten Interessensanstieg etwa ab Jahrgangsstufe 11. Gemäß dem Modell der Interessensdifferenzierung (Todt \& Schreiber, 1998) lässt sich der mittlere Interessensabfall auf einen Differenzierungsprozess zurückführen: Die Schülerinnen und Schüler fokussieren ihr Interesse nach Schuleintritt auf einige wenige Fächer, während die übrigen Fachinteressen sinken. Anhand der Fachinteressen Deutsch, Mathematik und Englisch zu Jahrgangsstufe 4 bis 11 wurde untersucht, inwieweit sich derartige Differenzierungsprozesse über eine latente Klassenanalyse mit längsschnittlichen Daten (LCA) nachzeichnen lassen. Die Analyse von $N=293$ Schülerinnen und Schülern bestätigte zunächst den Befund einer mittleren, kurvenlinearen Interessensentwicklung. Die LCA ergab fünf latente Klassen. Die Mittelwertdifferenzen der Fachinteressen ließen nur für zwei der fünf latenten Klassen eine zunehmende Interessensdifferenzierung zu Jahrgangsstufe 11 vermuten.
\end{abstract}

Schlüsselbegriffe: Interessensentwicklung, Fachinteressen, LCA mit längsschnittlichen Daten

\section{A Person Centered Approach for Analyzing the Development of Students'Subject-Interests in Language Arts, Mathematics, and English as a Foreign Language From Grade 4 to 11}

Summary: Numerous studies showed that students enter the school system with high and undifferentiated interests. However, academic interests on average decrease during the school course. Recent findings point to a stagnancy of this negative development in grade 7 and a slight increase in grade 11. According to the model of interest differentiation (Todt \& Schreiber, 1998) students' decreasing academic interests can be traced back to a process of differentiation: Students focus their interests on a limited number of subjects whereas their interests for the remaining subjects decrease. Using students' self-reports on their subject-interests in language arts, mathematics and English as a foreign language at grade 4 to grade 11 we analyzed whether (a) latent class(es) (latent class analysis with longitudinal data, LCA) of a differentiating interest profile can be found. On average the analysis of $N=293$ students showed decreasing interests up to grade 7 and slightly increasing interests in grade 11 . The LCA indicated five latent classes. Two of them revealed a pattern of interest differentiation in grade 11 .

Keywords: Interest development, subject-interests, latent class analysis with longitudinal data

\section{Autorenhinweis:}

Diese Veröffentlichung wurde ermöglicht durch eine Sachbeihilfe der Deutschen Forschungsgemeinschaft im Bamberger Forschungsprojekt Bildungsprozesse, Kompetenzentwicklung und Selektionsentscheidungen im Vorschul-und Schulalter (BiKS; Kennzeichen: WE 1478/4, AR 301/9 und AR 301/10) sowie im Folgeprojekt BiKS ${ }^{\text {plus[8-18] }}$ (Kennzeichen: AR 301/10-1).

Psychologie in Erziehung und Unterricht, 2017, 64, Preprint Online

DOI 10.2378/peu2017.art24d

(c) Ernst Reinhardt Verlag München Basel 
Bis heute ist die Frage, wie sich das Interesse von Schülerinnen und Schülern im Verlauf der Schulzeit entwickelt, eine der zentralen Fragen innerhalb der Interessensforschung. Während frühere Arbeiten vorrangig querschnittliche Studien berichten, wurden in den letzten Jahrzehnten mehr und mehr längsschnittliche Forschungsarbeiten veröffentlicht (Baumert \& Köller, 1998; Dotterer, McHale \& Crouter, 2009). Doch noch immer gibt es nur wenige Langzeitstudien, die eine Beobachtung der Interessensentwicklung über einen Großteil der gesamten Schullaufbahn ermöglichen. Darüber hinaus dominieren bisher Studien, welche - entsprechend eines variablenzentrierten Ansatzes - die mittleren Entwicklungen schulischer Interessen berichten. Im Sinne einer ontogenetischen Analyse der Interessensentwicklung ist jedoch ebenso eine intraindividuelle Betrachtung der Entwicklungsvorgänge vorzunehmen (vgl. Krapp, 2002). Um sowohl eine langzeitliche als auch eine personenzentrierte Betrachtung der Entwicklung schulischer Interessen zu ermöglichen, soll im vorliegenden Beitrag die Interessensentwicklung von Schülerinnen und Schülern in den Fächern Deutsch, Mathematik und Englisch vom Ende der Grundschule bis zum Beginn der Sekundarstufe II anhand einer latenten Klassenanalyse mit längsschnittlichen Daten untersucht werden.

\section{Theoretischer Hintergrund}

\section{Das Konzept des Fachinteresses}

Als theoretisches Rahmenmodell wird die Person-Gegenstands-Theorie des Interesses (Krapp, 1992, 2002) zugrunde gelegt. Demnach handelt es sich bei dem Interessenskonzept um eine spezifische Relation zwischen einer Person und einem diese Person interessierenden Gegenstand. Diese inhaltliche Bestimmtheit ist eines der zentralen Merkmale, welches das Interessenskonzept von anderen motivationalen Konstrukten unterscheidet. Strukturell umfasst ein Interesse (z. B. das Fachinteresse) nach Krapp (1992) drei Komponenten: Zum einen den Interessensgegenstand, welcher den konkreten In- halt des Interesses definiert (z. B. die Inhalte des Unterrichtsfachs Deutsch). Zweitens gibt es Interessenshandlungen, die zur Beschäftigung mit dem Interessensgegenstand vollzogen werden (z. B. ein Buch lesen oder einen Text schreiben). Und drittens konkrete Objekte, die herangezogen werden, um sich mit dem interessierenden Gegenstand auseinandersetzen zu können (z. B. ein Buch oder ein Gedicht).

Das Interessenskonzept zeichnet sich durch eine Wert- und eine emotionale Komponente aus: Die Person misst dem Interessensgegenstand einen persönlichen Wert zu und empfindet in der Summe der gegenstandsbezogenen Handlungen positive Emotionen bei der Beschäftigung mit dem Gegenstand (Krapp, 2002). Insbesondere mit Bezug auf die Wertkomponente wird zudem angenommen, dass sich Personen mit einem stabil entwickelten Interesse mit dem Interessensgegenstand identifizieren und dieser Teil ihrer Selbstdefinition wird (Krapp, 2002). Studien zum Interesse lassen sich danach unterscheiden, ob sie das situative oder individuelle Interesse von Personen analysieren. Während das situative Interesse nur in einer spezifischen, interessanten Situation vorhanden ist, handelt es sich bei dem individuellen Interesse um ein stabiles, im Selbstbild der Person verankertes Interesse (Krapp, 2002). In der vorliegenden Arbeit werden die Fachinteressen als individuelle Interessen gefasst. Gemäß Hoffmann (2002) kann das Fachinteresse auf zwei verschiedene Weisen konzipiert werden: Zum einen als ein Interesse an den Themen des jeweiligen Unterrichtsfachs, zum anderen als ein Interesse an dem gesamten Unterricht des Schulfaches - sowohl wie es gelehrt wird als auch was darin gelernt wird. Im vorliegenden Beitrag wird das Fachinteresse in dieser zweiten Konzeption gefasst.

\section{Die Entwicklung schulischer Interessen}

Ein zentraler und vielfach berichteter Befund der Interessensforschung verweist darauf, dass Schülerinnen und Schüler im Mittel mit einem undifferenziert hohen Interesse für die einzel- 
nen Schulfächer in die Schule eintreten und dieses Interesse im Verlauf der Schulzeit mehr und mehr zurückgeht (z. B. Baumert \& Köller, 1998; Dotterer et al., 2009; Frenzel, Goetz, Pekrun \& Watt, 2010; Frenzel, Pekrun, Dicke \& Goetz, 2012; Jacobs, Lanza, Osgood, Eccles \& Wigfield, 2002; Krapp, 2002; Potvin \& Hasni, 2014). Dieser Befund trifft für beide Geschlechter zu, wobei einzelne Studien auf leichte Geschlechtsunterschiede in den Verläufen verweisen. So berichtet etwa Helmke (1993), dass Jungen zwar mit höheren Interessen als Mädchen in die Schule eintreten, der Interessensabfall bis Jahrgangsstufe 5 bei Jungen jedoch steiler verläuft als bei Mädchen. Mädchen zeigen demnach einen flacheren Interessensabstieg als Jungen und ein stagnierendes Interesse im späteren Schulverlauf (siehe auch Dotterer et al., 2009).

Ansätze zur Erklärung des negativen Entwicklungstrends schulischer Interessen verweisen einerseits auf die außerschulischen Interessensbereiche der Jugendlichen, welche im zunehmenden Maße mit den schulischen Interessen konkurrieren (z. B. Hartinger \& FöllingAlbers, 2002). Andererseits verweist der StageEnvironment-Fit-Ansatz (Eccles et al., 1993) auf eine zunehmende fehlende Passung zwischen den Bedürfnissen und Interessen der Jugendlichen und den Angebotsstrukturen des schulischen Kontexts. Darüber hinaus kann gemäß des Modells der Interessensdifferenzierung angenommen werden, dass es sich bei dem mittleren Interessensabfall innerhalb einzelner schulischer Fachbereiche um einen funktionalen Prozess der Selbstdifferenzierung handelt (Baumert \& Köller, 1998; Daniels, 2008; Todt \& Schreiber, 1998). Demnach treten Schülerinnen und Schüler zwar mit undifferenziert hohen Interessen für sämtliche Schulfächer in die Schule ein. Im Verlauf der Schulzeit tritt jedoch ein Prozess der Differenzierung und Fokussierung auf einige wenige Interessensbereiche ein. Während also die Interessen für die Mehrzahl der schulischen Fachbereiche absinken, wird angenommen, dass das Interesse für einige wenige, individuell ausgewählte Fachbereiche stabil bleibt. Bei einer Betrachtung mittlerer Entwick- lungsverläufe bleiben derartige intraindividuelle Prozesse jedoch verborgen (Daniels, 2008). Insbesondere Studien zur Differenzierung des akademischen Selbstkonzepts verweisen darauf, dass der Prozess der Selbstdifferenzierung vorrangig zwischen Fächern der verbalen Domäne einerseits und der mathematischen Domäne andererseits zu erwarten ist (Dickhäuser, 2003; Möller \& Marsh, 2013; Pohlmann, 2005). Diese Befunde konnten bereits auf das Fachinteresse übertragen werden, allerdings verweisen die bisherigen Ergebnisse auf eine vollständige $\mathrm{Me}$ diation des Leistungseffekts auf das Fachinteresse über das fachspezifische Selbstkonzept (Pohlmann, 2005; Schurtz, Pfost, Nagengast \& Artelt, 2014). Um den Prozess der zunehmenden Interessensdifferenzierung im Schulverlauf empirisch nachzeichnen zu können, bedarf es jedoch sowohl Langzeituntersuchungen über die gesamte Schulzeit hinweg als auch einer gleichzeitigen Betrachtung der Interessensentwicklung innerhalb mehrerer schulischer Fachbereiche.

\section{Forschungsstand zur Differenzierung der Interessen im Schulverlauf}

Einige wenige Studien verfolgen die mittlere Interessensentwicklung der Schülerinnen und Schüler von der frühen bis zur späten Schulzeit. Diese Arbeiten verweisen auf eine Stagnation des Interessensabfalls im späteren Verlauf der Sekundarstufe oder sogar einen leichten Wiederanstieg des schulischen Interesses ab der Jahrgangsstufe 11 bzw. ab dem Alter von etwa 17 Jahren (Dotterer et al., 2009; Frenzel et al., 2010; Jacobs et al., 2002). Daniels (2008) berichtet die Korrelationswerte zwischen den Fachinteressen als ein Maß der Interessensdifferenzierung. Demnach nehmen die Korrelationen zwischen den Fachinteressen Physik und Biologie von Jahrgangsstufe 7 bis $10 \mathrm{ab}$, was die Autorin als ein Zeichen der zunehmenden Unabhängigkeit und somit Interessensdifferenzierung deutet (siehe auch Baumert \& Köller, 1998). Allerdings sind sowohl die Betrachtung von Mittelwerten als auch die Betrachtung von Korrelationswerten innerhalb einer Gesamtstichprobe ungeeignet, um den in- 
traindividuellen Prozess der Differenzierung verschiedener Interessensbereiche nachzuzeichnen. Vielmehr bedarf es hierfür einen personenzentrierten Analyseansatz. Archambault, Eccles und Vida (2010) verfolgen einen solchen und berichten sieben verschiedene Profile der Interessens- und Selbstkonzeptentwicklung im sprachlichen Bereich von Jahrgangsstufe 1 bis 12. Demnach zeigen nur zwei Profile ein kontinuierlich absinkendes Interesse für den gesamten Beobachtungszeitraum. Die übrigen Profile verweisen auf eine Stagnation des negativen Trends bzw. einen Wiederanstieg des Interesses und Selbstkonzepts ab Jahrgangsstufe 7. Auch MusuGillette, Wigfield, Harring und Eccles (2015) verfolgen einen personenzentrierten Ansatz bei der Analyse der Entwicklung des Selbstkonzeptes, der Wertschätzung und des Interesses im Fach Mathematik von Jahrgangsstufe 4 bis zum Collegeeintritt. Die Verlaufsanalysen zum Mathematikinteresse verweisen auf drei latente Klassen. Alle drei Profile zeigen eine kurvenlineare Entwicklung des Interesses. Sie variieren lediglich bezüglich der Steilheit des Interessensabfalls sowie des Zeitpunktes der Stagnation des Interessensabfalls bzw. des Wiederanstieges des Interesses im späteren Schulverlauf. Bisher fehlt es jedoch an theoretischen Erklärungsansätzen für diese Befunde einer kurvenlinearen Interessensentwicklung. Darüber hinaus betrachten diese Studien ausschließlich einzelne schulische Interessensbereiche. Ein Prozess der intraindividuellen Differenzierung zwischen mehreren Interessensbereichen kann somit von diesen Studien nicht abgebildet werden. Eine erste, personenzentrierte Betrachtung der gemeinsamen Entwicklung schulischer Interessen in den Fächern Deutsch, Mathematik und Englisch findet sich in dem Beitrag von Schurtz \& Artelt (2014). Unter Verwendung der Daten der BiKS-8-14Studie (Bildungsprozesse, Kompetenzentwicklung und Selektionsentscheidungen im Vorschul- und Schulalter) berichten die Autoren fünf latente Klassen mit unterschiedlichen Profilen der Interessensentwicklung von Jahrgangsstufe 4 bis 7 . Das Fachinteresse Englisch ging allerdings erst ab der Jahrgangsstufe 5 in die Analysen mit ein, da es erst in der Sekundarstufe mit einem einheitlich verbindenden Curriculum gelehrt und benotet wird. Hierbei zeigen alle fünf latenten Klassen einen stabilen oder absinkenden Verlauf der Interessensentwicklung. Die Profile von zwei latenten Klassen verweisen auf eine undifferenzierte Entwicklung der Interessen in den Fächern Deutsch, Mathematik und Englisch auf einem hohen bzw. niedrigen Niveau. Die übrigen latenten Klassen wiesen während der Grundschulzeit einen stärkeren Grad der Differenzierung als im Verlauf der Sekundarstufe auf. Die Befunde dieser Studie stellen demnach die These einer zunehmenden Differenzierung schulischer Interessen im Schulverlauf infrage. Allerdings beschränken sich diese Analysen auf den Zeitraum von Jahrgangsstufe 4 bis 7, sodass offen bleibt, ob eventuell im weiteren Verlauf der Sekundarstufe ein (erneuter) Prozess der Differenzierung einsetzt.

\section{Forschungsfragen}

Im vorliegenden Beitrag soll die Interessensentwicklung von Schülerinnen und Schülern in den Fächern Deutsch, Mathematik und Englisch von Jahrgangsstufe 4 bis 11 untersucht werden. Über eine Betrachtung mittlerer Entwicklungstendenzen hinaus wird anhand der Methode der latenten Klassenanalyse der Frage nachgegangen, ob sich Subgruppen von Schülerinnen und Schülern mit differenziellen strukturellen Verläufen der Interessensentwicklung nachzeichnen lassen (vgl. Schurtz \& Artelt, 2014). Hierbei soll insbesondere der Frage nachgegangen werden, ob sich ein Profil der zunehmenden Interessensdifferenzierung aufzeigen lässt. Zudem wird untersucht, ob sich die in der Literatur berichtete leicht positive Entwicklung schulischer Interessen am Ende der Sekundarstufe I bzw. zu Beginn der Sekundarstufe II sowohl im mittleren Verlauf als auch innerhalb der einzelnen latenten Klassen replizieren lässt (siehe z. B. Dotterer et al., 2009). Als Datengrundlage verwenden wir die BiKS-8-14-Studie und analysieren dieselben Messzeitpunkte wie bei Schurtz und Artelt (2014) unter Ergänzung der 
Jahrgangsstufen 9 und 11. Hierfür wird zudem auf Daten des Folgeprojektes BiKS ${ }^{\text {plus }[8-18]}$ zurückgegriffen. Um sicherzustellen, dass alle Schülerinnen und Schüler eine vergleichbare Konzeption des Englischunterrichts einschätzen, wird das Fachinteresse Englisch erst ab der Jahrgangsstufe 5 mit in die Analysen aufgenommen (vgl. Schurtz \& Artelt, 2014). Mit Bezug auf die bisherigen Befunde von Schurtz und Artelt (2014) gehen wir davon aus, dass sich bis zur Jahrgangsstufe 7 keine Differenzierungsprozesse zwischen den analysierten Fachinteressen aufzeigen - sowohl mit Bezug auf die mittleren als auch personenzentrierten Analysen. Ab Jahrgangsstufe 7 vermuten wir, dass sich im Mittel sowie für mindestens eine der latenten Klassen ein leicht positiver Trend der Interessensentwicklung zeigt (siehe z. B. Dotterer et al., 2009). Offen bleibt, ob sich eine oder mehrere latente Klassen ergeben, deren Profile auf einen Prozess der zunehmenden Interessensdifferenzierung im späteren Schulverlauf verweisen.

\section{Methode}

\section{Stichprobe}

Zur Analyse der dargestellten Forschungsfragen verwenden wir Daten der BiKS-8-14-Studie sowie des Folgeprojekts BiKS ${ }^{\text {plus[8-18] }}$. Das von der Deutschen Forschungsgemeinschaft (DFG) geförderte Projekt BiKS (Bildungsprozesse, Kompetenzentwicklung und Selektionsentscheidungen im Vorschul-und Schulalter) lief von 2005 bis 2011 und besteht aus zwei separaten Längsschnitten. Innerhalb der BiKS-8-14-Studie wurden Schülerinnen und Schüler aus Bayern und Hessen von Jahrgangsstufe 3 bis 9 verfolgt. Während in der Grundschule eine halbjährliche Messung erfolgte, wurden die Schülerinnen und Schüler ab der Sekundarstufe I im jährlichen Abstand befragt. Um diesen Längsschnitt auch über die Jahrgangsstufe 9 hinaus weiterverfolgen zu können, wurde eine weitere DFG-Finanzierung beantragt. Im Jahr 2013 startete das neue Projekt BiKS ${ }^{\text {plus[8-18]. Hierbei wurden }}$ die Schülerinnen und Schüler aus der BiKS-8-14Studie gebeten, für weitere drei Jahre an dem BiKSProjekt teilzunehmen. Die im Folgenden verwendete Stichprobe umfasst Schülerinnen und Schüler, die seit der vierten Jahrgangsstufe an der BiKS-8-14-
Studie teilgenommen haben $(N=2157)$. Ausgeschlossen wurden diejenigen Fälle, die in der fünften Jahrgangsstufe eine andere Fremdsprache als Englisch erlernten $(n=96)$. Ebenso wurden Fälle ausgeschlossen, die im Jahr 2014 - der ersten Erhebungswelle von BiKS ${ }^{\text {plus[8-18] }}$ - nicht die elfte Jahrgangsstufe des allgemeinbildenden Schulsystems besuchten $(n=1768)$. Demnach konnten die Daten von $N=293$ Schülerinnen und Schülern ( $45,4 \%$ weiblich) in die Analysen aufgenommen werden, welche am Ende der elften Jahrgangsstufe mehrheitlich das Gymnasium besuchten $(85 \%$ der Schülerinnen und Schüler). In Jahrgangsstufe 5 lag das mittlere Alter bei 11.4 Jahren $(S D=0.4)$ und 15,6\% der Schülerinnen und Schüler lebten in einem Haushalt mit Migrationshintergrund, das heißt ein oder beide Elternteile wurden im Ausland geboren.

\section{Instrumente}

Die Interessen der Schülerinnen und Schüler an den Fächern Deutsch, Mathematik und Englisch wurden zu den Messzeitpunkten in Jahrgangsstufe 4 bis 9 anhand einer schriftlichen Befragung über jeweils vier Items erfasst (adaptiert nach Bildungsverläufe und psychosoziale Entwicklung im Jugend- und jungen Erwachsenenalter [BIJU]; Baumert, Gruehn, Heyn, Köller \& Schnabel, 1997). Zwei dieser Items erfassten die kognitiv-wertbezogene Komponente des Interessengegenstandes (,Wie viel liegt dir daran, den Stoff des Faches Deutsch zu behalten?“; „Wie viel liegt dir daran, im Fach Deutsch viel zu wissen?"). Ein Item zielte auf die emotionale Komponente (,Wie sehr freust du dich auf eine Stunde im Fach Deutsch?") und ein weiteres Item erfasste, inwieweit sich die Schülerinnen und Schüler auch in Zukunft mit dem Interessensgegenstand beschäftigen möchten („Wie gerne würdest du im Fach Deutsch noch mehr Stunden haben als bisher?"). Die Beantwortung der Items erfolgte über eine fünfstufige Antwortskala ( $g a r$ nicht; wenig; mittel; ziemlich; sehr). In Jahrgangsstufe 11 erfolgte die Datenerfassung im Rahmen von BiKS ${ }^{\text {plus[8-18] }}$ anhand eines telefonischen Interviews. Um einen guten Interviewfluss zu gewährleisten, wurde die Struktur der Frage leicht abgewandelt. Die Schülerinnen und Schüler wurden gebeten, auf einer Skala von $1=$ gar nicht bis $5=$ seh $r$ die folgenden Fragen mit Bezug auf die Fächer Deutsch, Mathematik und Englisch zu beantworten: „Wie viel liegt Ihnen daran, den Stoff der folgenden Fächer zu behalten?"; „Wie viel liegt Ihnen daran, in den folgenden Fächern 
viel zu wissen?"; „Wie sehr freuen Sie sich auf eine Stunde in den folgenden Fächern?" und „Wie gerne würden Sie in den folgenden Fächern noch mehr Stunden haben als bisher?" Die Reliabilitäten der einzelnen Skalen waren zu allen Messzeitpunkten zufriedenstellend (Deutsch: Cronbachs $\alpha=.81-.87$; Mathematik: Cronbachs $\alpha=.83-.88$; Englisch: Cronbachs $\alpha=.80-.85$ ).

\section{Analysemethode}

Die Entwicklung der schulischen Interessen in den Fächern Deutsch, Mathematik und Englisch wurde zunächst in Anlehnung an bisherige Studien über die Betrachtung von mittleren Verläufen analysiert. Neben der Untersuchung von Mittelwertveränderungen über die Zeit hinweg wurden hierbei ergänzend auch die Korrelationen zwischen den Schulfächern am jeweiligen ersten und letzten Erhebungszeitpunkt betrachtet. In der Literatur werden abnehmende Korrelationen im Zeitverlauf als Anzeichen für eine zunehmende Differenzierung angesehen (vgl. Baumert \& Köller, 1998; Daniels, 2008). Hierbei verweist auch die Veränderung von einer positiv- zu einer negativ-signifikanten Korrelation auf eine zunehmende Differenzierung der jeweiligen Fachinteressen. Ansteigende Korrelationen deuten hingegen auf einen zunehmenden Entwicklungszusammenhang und damit auf zunehmend undifferenzierte Fächerpaare hin. Als eine Erweiterung bisheriger Studien wurde die Veränderung der Korrelationen im vorliegenden Beitrag auf Signifikanz geprüft. Hierfür wurden die Korrelationen in Fishers Z-Werte umgewandelt und anschließend die $95 \%$-Konfidenzintervalle der Korrelationen berechnet (Bühner \& Ziegler, 2009). Um der Frage nachzugehen, ob sich Personengruppen mit differenziellen Verläufen der Interessensentwicklung aufzeigen lassen, wurde anschließend eine latente Klassenanalyse mit längsschnittlichen Daten unter Verwendung von Mplus durchgeführt (Muthén \& Muthén, 1998-2010). Hierbei gingen die Skalen der Fachinteressen Deutsch und Mathematik von der ersten und zweiten Hälfte der Jahrgangsstufe 4 sowie die Skalen der Fachinteressen Deutsch, Mathematik und Englisch von Jahrgangsstufe 5, 6, 7, 9 und 11 in die Analysen ein. Die zu analysierende Fallzahl von $n=293$ Fällen stellt zwar eine kleine Stichprobe dar, erfüllt jedoch die Mindestanforderungen zur Durchführung einer LCA (vgl. Nylund, Asparouhov \& Muthén, 2007). Zudem verweisen die Befunde von Kim (2012) darauf, dass eine geringe Stichproben- größe zum Teil durch eine hohe Anzahl an Indikatoren, die über einen langen Zeitraum hinweg gesammelt wurden, ausgeglichen werden kann. Studien zu alternativen Analysemethoden wie beispielsweise Growth-Mixture-Modellen (Muthén, 2001) verweisen bisher auf widersprüchliche Befunde hinsichtlich der Eignung für derartig geringe Fallzahlen (siehe Martin \& von Oertzen, 2015; Peugh \& Fan, 2012). Vor dem Hintergrund dieser Befundlage erscheint die Durchführung einer LCA trotz der geringen Fallzahl als angemessen.

Die Anteile fehlender Werte der Skalen zum Fachinteresse liegen in den unterschiedlichen Jahrgangsstufen zwischen $0 \%$ (Fachinteresse Deutsch in Jahrgangsstufe 11) und 8,5\% (Fachinteresse Englisch in Jahrgangsstufe 5). Eine Ausnahme bezieht sich auf die drei Interessensskalen in der neunten Jahrgangsstufe, die mit 25,3\% deutlich höher liegen. ${ }^{1}$ Für einen adäquaten Umgang mit den fehlenden Werten wurde der FIML (Full Information Maximum Likelihood)Algorithmus verwendet. Die latente Klassenanalyse mit längsschnittlichen Daten wurde für eine bis zehn latente Klassen durchgeführt. Um die endgültige Anzahl latenter Klassen auszuwählen, wurden verschiedene statistische Kennwerte herangezogen. Als Informationskriterien wurden der AIC (Akaike Information Criterion), BIC (Bayesian Information Criterion) und der adjusted BIC ausgewertet. Je geringer diese Indikatoren ausfallen, umso besser ist die Modellanpassung. Darüber hinaus wurden als inferenzstatistische Entscheidungskriterien der Vuong-LoMendell-Rubin Likelihood Ratio Test (LMR-Test; Lo, Mendell \& Rubin, 2001; Vuong, 1989) sowie der Parametric Bootstrapped Likelihood Ratio Test (BLRT; McLachlan \& Peel, 2000) ausgewertet. Beide testen, inwieweit die Lösung mit k-latenten Klassen gegenüber derjenigen mit k-1-latenten Klassen einen besseren Modellfit bietet. Ein signifikanter $p$-Wert

1 Der erhöhte Anteil fehlender Werte lässt sich auf einen Einbruch der Teilnehmerzahlen im Projekt BiKS-8-14 in Jahrgangsstufe 8 zurückführen. Zu diesem Zeitpunkt forderte das Kultusministerium eine erneute schriftliche Einverständniserklärung von allen Teilnehmerinnen und Teilnehmern sowie deren Eltern. Dies führte leider zu stark erhöhten Ausfällen, welche sich auch in Jahrgangsstufe 9 fortsetzten. Für die Stichprobenrekrutierung des Folgeprojektes BiKS ${ }^{\text {plus[8-18] }}$ wurden sämtliche ehemaligen Teilnehmerinnen und Teilnehmer des Projektes BiKS-8-14 über das Folgeprojekt informiert und um ihre erneute Teilnahme gebeten. Auf diese Weise konnten Schülerinnen und Schüler im Rahmen des Folgeprojektes befragt werden, welche in Jahrgangsstufe 9 nicht teilgenommen hatten. 
bevorzugt hierbei die Lösung mit k-latenten Klassen gegenüber derjenigen mit k-1-latenten Klassen (Asparouhov \& Muthén, 2012; Nylund et al., 2007). Schließlich wurde noch auf das Maß der Entropie zurückgegriffen, welches die Klassifikationsgenauigkeit der jeweiligen Lösung latenter Klassen auf einer Skala zwischen 0 und 1 abbildet. Je höher der Entropie-Wert, umso genauer erfolgte die Klassifikation der Fälle bei der jeweiligen Anzahl latenter Klassen (Wang \& Wang, 2012). Hierbei ist eine Entropie mit einem Wert über .70 zu bevorzugen (z. B. Meeus, Van de Schoot, Klimstra \& Branje, 2011). Allerdings stellen die statistischen Kennwerte nur Anhaltspunkte zur Auswahl der endgültigen Anzahl latenter Klassen dar. So ist beispielsweise bei den Informationskriterien fraglich, ab welcher Differenzgröße ein Modell dem anderen vorzuziehen ist. Die letztendliche Entscheidung für oder gegen eine Lösung sollte daher aus inhaltlich-theoretischen Gesichtspunkten erfolgen (vgl. Alexandrowicz, 2008). Für den vorliegenden Beitrag war hierbei entscheidend, ob eine Lösung mit größerer Anzahl latenter Klassen im Vergleich zu den Lösungen mit geringerer Klassenzahl zusätzliche strukturelle Verläufe der Interessensentwicklung aufzeigen. Abschließend erfolgte die Charakterisierung der verschiedenen Profile der Interessensentwicklung. Hierbei wurde zwischen (annähernd) differenzierten und (annähernd) undifferenzierten Verläufen der Interessensentwicklung unterschieden. Als (annähernd) differenziert gelten zwei Fachinteressen zu dem jeweiligen Messzeitpunkt, wenn sich die auf Grundlage der Standardfehler berechneten Konfidenzintervalle der Mittelwerte (nur knapp) nicht überschneiden $\left(\mathrm{KI}=M+/-1.96^{*} S E\right)$. Bei einer (nur knappen) Überschneidung der Konfidenzintervalle gelten die beiden Fachinteressen zu dem jeweiligen Messzeitpunkt als (annähernd) undifferenziert. Als ergänzendes Maß für die Differenzierung der Fachinteressen wurden für die einzelnen latenten Klassen ebenfalls die bivariaten Korrelationen zum jeweiligen ersten und letzten Messzeitpunkt berechnet.

\section{Ergebnisse}

\section{Betrachtung der mittleren Entwicklungsverläufe}

Tabelle 1 zeigt die Mittelwerte und Standardfehler der Fachinteressen Deutsch, Mathematik und Englisch zu den Messzeitpunkten, welche für die latente Klassenanalyse verwendet wurden. Für die Fachinteressen Deutsch und Mathematik zeigt sich für den Zeitraum zwischen dem ersten Halbjahr der vierten bis zur neunten Jahrgangsstufe ein negativer Entwicklungstrend. Für das Fachinteresse Englisch zeigt sich dieser entsprechend für die fünfte bis neunte Jahrgangsstufe. Alle drei Fachinteressen verzeichnen einen leichten Interessensanstieg zu Jahrgangsstufe 11. Im Fach Englisch erreicht das mittlere Interesse sogar den Anfangswert aus Jahrgangsstufe 5. Unter Verwendung der bisherigen mittleren Analysemethoden der Interessensdifferenzierung verweist der Vergleich der Konfidenzintervalle der mittleren Fachinteressen Deutsch und Mathematik auf zunehmend undifferenzierte Interessen: Während die Mittelwerte der beiden Fachinteressen im ersten Halbjahr der vierten Jahrgangsstufe signifikant verschieden sind, kommt es in Jahrgangsstufe $11 \mathrm{zu}$ einer Überschneidung der Konfidenzintervalle.

Tab. 1: Mittelwert und Standardfehler der Fachinteressen Deutsch, Englisch und Mathematik innerhalb der Gesamtstichprobe $(N=293)$

\begin{tabular}{|l|c|c|c|c|c|c|}
\hline & \multicolumn{2}{|c|}{ Fachinteresse Deutsch } & \multicolumn{2}{c|}{ Fachinteresse Mathematik } & \multicolumn{2}{c|}{ Fachinteresse Englisch } \\
\hline Jg. & $\boldsymbol{M}(\mathrm{SE})$ & $\mathbf{N}$ & $\boldsymbol{M}(\mathrm{SE})$ & $\mathbf{N}$ & $\boldsymbol{M}(\mathrm{SE})$ & $\boldsymbol{N}$ \\
\hline Jg. 4.1 & $3.66(.06)$ & 272 & $4.06(.06)$ & 272 & - & - \\
\hline Jg. 4.2 & $3.61(.06)$ & 282 & $4.01(.06)$ & 282 & - & - \\
\hline Jg. 5 & $3.10(.06)$ & 269 & $3.41(.06)$ & 269 & $3.58(.06)$ & 268 \\
\hline Jg. 6 & $2.79(.05)$ & 269 & $3.14(.06)$ & 269 & $3.37(.06)$ & 269 \\
\hline Jg. 7 & $2.78(.05)$ & 290 & $3.11(.06)$ & 290 & $3.34(.05)$ & 290 \\
Jg. 9 & $2.86(.06)$ & 219 & $3.06(.06)$ & 219 & $3.26(.06)$ & 219 \\
\hline Jg. 11 & $3.18(.05)$ & 293 & $3.28(.05)$ & 291 & $3.59(.05)$ & 284 \\
\hline
\end{tabular}

Anmerkungen: Jg. = Jahrgangsstufe; Jg. 4.1 = erstes Halbjahr der Jg. 4; Jg. 4.2 = zweites Halbjahr der Jg. 4. 
Allerdings ergeben die bivariaten Korrelationen beider Messzeitpunkte keinen signifikanten $\mathrm{Zu}-$ sammenhang, Jahrgangsstufe 4.1: $r=.08(-.04$; $.19), p>.05$; Jahrgangsstufe $11: r=.02(-.10$; $.13), p>.05$. Für die Fachinteressen Deutsch und Englisch zeigen sich für Jahrgangsstufe 5 und 11 signifikante Mittelwertunterschiede, sodass der mittlere Verlauf beider Fachinteressen unter Verwendung der bisherigen Analysemethoden der Interessensdifferenzierung als stabil differenziert bezeichnet werden kann. Die bivariaten Korrelationen in Jahrgangsstufe 5 und 11 verweisen zwar auf einen leicht sinkenden Zusammenhang beider Fachinteressen, allerdings überschneiden sich die berechneten Konfidenzintervalle, Jahrgangsstufe $5: r=.35(.24 ; .45), p<.05$; Jahrgangsstufe 11 : $r=.21(.09 ; .32), p<.05$. Folglich verweisen die Korrelationswerte auf einen stabilen, positiven Entwicklungszusammenhang der Fachinteressen Deutsch und Englisch innerhalb der Gesamtstichprobe. Schließlich zeigt sich bei Verwendung der gängigen Analyseart der Interessensdifferenzierung für das mittlere Fachinteresse Englisch und Mathematik ein Muster der zunehmenden Differenzierung: Während in Jahrgangsstufe 5 keine signifikanten Mittelwertunterschiede vorliegen, sind die Mittelwerte beider Fachinteressen in Jahrgangsstufe 11 signifikant verschieden. Die Korrelationen verweisen ebenfalls auf einen signifikanten, abnehmenden Zusammenhang, welcher in Jahrgangsstufe 11 negativ ausfällt, Jahrgangsstufe $5: r=.17(.05 ; .29), p<.05$; Jahrgangsstufe 11 : $r=-.15(-.26 ;-.03), p<.05$.

\section{Ergebnisse der latenten Klassenanalyse}

Die latente Klassenanalyse mit längsschnittlichen Daten konnte für eine bis sieben latente Klassen durchgeführt werden. Ab einer Anzahl von acht latenten Klassen konnte der beste Loglikelihood-Wert des Modells nicht mehr spezifiziert werden. Dies spricht dafür, dass die Daten keine Anzeichen für entsprechend hohe Zahlen latenter Klassen aufweisen (Muthén, 2007). Die statistischen Kennwerte der latenten Klassenanalyse werden daher für eine bis sieben latente Klassen in Tabelle 2 angegeben. Die Informationskriterien AIC und adjusted BIC werden mit zunehmender Anzahl latenter Klassen durchweg kleiner, sodass sie auf keine spezifische Lösung verweisen. Der BIC nimmt hingegen bei der Lösung mit vier und fünf latenten Klassen den geringsten und nahezu gleich hohen Wert an, sodass beide Lösungen favorisiert werden. Der LMR-Test nimmt lediglich bei der Lösung mit zwei latenten Klassen einen signifikanten Wert an und verweist somit darauf, dass eine Zweiklassenlösung besser zu den Daten passt als eine Lösung mit nur einer latenten Klasse.

Der BLRT nimmt für alle Zahlen latenter Klassen signifikante Werte an, sodass dieser Kennwert keine Informationen zur Klassenauswahl liefert. Schließlich zeigt sich mit Bezug auf die Entropie, dass die Werte für drei bis sieben latente Klassen zwischen .81 und .86 streuen und somit ähnlich hoch ausfallen. Mit Bezug auf die statistischen Kennwerte werden somit

Tab. 2: Statistische Kennwerte der latenten Klassenanalyse mit längsschnittlichen Daten für ein bis sieben latente Klassen

\begin{tabular}{|l|c|c|c|c|c|c|}
\hline $\mathbf{k}$ & AIC & BIC & Adj. BIC & LMRT & BLRT & Entropie \\
\hline $\mathbf{1}$ & 13685.72 & 13825.57 & 13705.06 & - & - & - \\
\hline $\mathbf{2}$ & 13316.58 & 13530.03 & 13346.10 & $-6804.861^{*}$ & $-6804.861^{*}$ & .77 \\
\hline 3 & 13136.42 & 13423.48 & 13176.12 & -6600.29 & $-6600.29^{*}$ & .81 \\
\hline 4 & 13033.70 & 13394.36 & 13083.58 & -6490.21 & $-6490.21^{*}$ & .83 \\
\hline $\mathbf{5}$ & $\mathbf{1 2 9 6 4 . 0 9}$ & 13398.35 & 13024.14 & -6418.85 & $-6418.85^{*}$ & .82 \\
$\mathbf{6}$ & 12910.18 & 13418.05 & 12980.41 & -6382.05 & $-6382.05^{*}$ & .84 \\
\hline 7 & 12869.09 & 13450.56 & 12949.50 & -6352.79 & $-6352.79^{*}$ & .86 \\
\hline
\end{tabular}

Anmerkungen: Die endgültig ausgewählte Lösung latenter Klassen ist fett markiert. $\mathrm{k}=$ Anzahl latenter Klassen; AIC = Akaike Information Criterion, $\mathrm{BIC}=$ Bayesian Information Criterion, Adj. BIC $=$ adjusted Bayesian Information Criterion, LMRT = Vuong-Lo-Mendell-Rubin Likelihood Ratio Test, BLRT $=$ Parametric Bootstrapped Likelihood Ratio Test. 
die Lösungen mit zwei, vier und fünf latenten Klassen leicht bevorzugt. Die Auswahl der Anzahl latenter Klassen aus inhaltlich-theoretischen Gesichtspunkten heraus favorisiert schließlich die Lösung mit fünf latenten Klassen. Im Vergleich zu den Lösungen mit zwei sowie vier latenten Klassen zeigen sich bei der Lösung mit fünf latenten Klassen mehrere Profile mit neuen strukturellen Verläufen der Interessensentwicklung.

\section{Charakterisierung der Lösung mit fünf latenten Klassen}

Die Mittelwerte und Standardabweichungen der schulfachbezogenen Interessen innerhalb der fünf latenten Klassen finden sich in Tabelle 3. Für eine bessere Anschaulichkeit der Entwicklungsverläufe sind die Mittelwerte der Fachinteressen noch einmal grafisch in Abbildung 1 dargestellt.
Die bivariaten Korrelationen der Fachinteressen zu den jeweils ersten und letzten Erhebungszeitpunkten sind in Tabelle 4 abgebildet.

Das Entwicklungsprofil der Fachinteressen innerhalb der latenten Klasse eins $(n=63$, $58,7 \%$ weiblich) lässt sich anhand der Mittelwertvergleiche bis zur Jahrgangsstufe 9 als stabil undifferenziert beschreiben. Die Fachinteressen verlaufen auf einem mittleren Niveau, wobei das Fachinteresse Englisch leicht höhere Werte als das Fachinteresse Deutsch und dieses wiederum höhere Werte als das Fachinteresse Mathematik aufweist. In Jahrgangsstufe 11 zeigt sich ein signifikanter Mittelwertunterschied für das Fachinteresse Englisch und Mathematik, sodass hier von einer zunehmenden Differenzierung im Zeitverlauf ausgegangen werden kann. Die Betrachtung der Korrelationen ergibt für diese latente Klasse keine signifikanten Werte. Auch die Mittelwertvergleiche der latenten Klasse

Tab. 3: Mittelwerte und Standardfehler der Fachinteressen Deutsch, Mathematik und Englisch innerhalb der fünf latenten Klassen

\begin{tabular}{|c|c|c|c|c|c|c|c|}
\hline & Jg. 4.1 & Jg. 4.2 & $\mathrm{Jg} \cdot 5$ & Jg. 6 & Jg. 7 & Jg. 9 & Jg. 11 \\
\hline \multicolumn{8}{|c|}{ Latente Klasse $1(n=63)$} \\
\hline FI De & $3.86(.23)$ & $3.91(.17)$ & $3.68(.18)$ & $3.21(.16)$ & $3.19(.16)$ & $3.63(.22)$ & $3.71(.16)$ \\
\hline $\mathrm{FI} \mathrm{Ma}$ & $3.35(.21)$ & $3.26(.16)$ & $3.17(.22)$ & $2.91(.16)$ & $3.02(.17)$ & $2.97(.22)$ & $3.15(.19)$ \\
\hline FI Eng & - & - & $3.84(.14)$ & $3.58(.17)$ & $3.51(.19)$ & $3.58(.23)$ & $3.94(.15)$ \\
\hline \multicolumn{8}{|c|}{ Latente Klasse $2(n=63)$} \\
\hline $\mathrm{FIDe}$ & $4.10(.17)$ & $3.98(.22)$ & $2.83(.16)$ & $2.51(.15)$ & $2.31(.18)$ & $2.41(.16)$ & $2.88(.20)$ \\
\hline $\mathrm{FI} \mathrm{Ma}$ & $4.22(.27)$ & $4.12(.23)$ & $2.97(.22)$ & $2.78(.26)$ & $2.50(.21)$ & $2.20(.20)$ & $2.77(.13)$ \\
\hline FI Eng & - & - & $3.29(.20)$ & $3.11(.18)$ & $2.87(.24)$ & $2.86(.20)$ & $3.69(.19)$ \\
\hline \multicolumn{8}{|c|}{ Latente Klasse $3(n=79)$} \\
\hline FI De & $3.92(.18)$ & $3.96(.20)$ & $3.54(.21)$ & $3.17(.18)$ & $3.24(.22)$ & $3.16(.21)$ & $3.27(.20)$ \\
\hline $\mathrm{FI} \mathrm{Ma}$ & 4.55 (.09) & $4.67(.08)$ & $4.02(.15)$ & $3.74(.18)$ & $3.64(.15)$ & $3.58(.15)$ & $3.63(.15)$ \\
\hline FI Eng & - & - & $4.16(.15)$ & $4.00(.20)$ & $3.84(.15)$ & $3.72(.15)$ & $3.68(.10)$ \\
\hline \multicolumn{8}{|c|}{ Latente Klasse $4(n=59)$} \\
\hline FI De & $2.84(.45)$ & $2.85(.42)$ & $2.45(.22)$ & $2.39(.26)$ & $2.36(.38)$ & $2.41(.39)$ & $2.84(.43)$ \\
\hline $\mathrm{FI} \mathrm{Ma}$ & $4.58(.28)$ & $4.60(.16)$ & $3.81(.24)$ & $3.48(.30)$ & $3.51(.22)$ & $3.59(.22)$ & $3.67(.18)$ \\
\hline FI Eng & - & - & $3.17(.22)$ & $2.90(.20)$ & $3.15(.19)$ & $2.84(.18)$ & $3.06(.21)$ \\
\hline \multicolumn{8}{|c|}{ Latente Klasse $5(n=29)$} \\
\hline FI De & $3.03(.24)$ & $2.58(.30)$ & $2.65(.19)$ & $2.32(.15)$ & $2.47(.18)$ & $2.49(.24)$ & $3.07(.17)$ \\
\hline $\mathrm{FI} \mathrm{Ma}$ & $2.88(.30)$ & $2.32(.24)$ & $2.57(.20)$ & $2.22(.16)$ & $2.40(.13)$ & $2.70(.26)$ & $2.91(.17)$ \\
\hline FI Eng & - & - & $2.94(.29)$ & $2.74(.21)$ & $3.05(.25)$ & $3.14(.21)$ & $3.37(.20)$ \\
\hline
\end{tabular}

Anmerkungen: Standardfehler stehen in Klammern; FI = Fachinteresse; De = Deutsch, Ma = Mathematik, Eng = Englisch; Jg. = Jahrgangsstufe; Jg. 4.1 = erstes Halbjahr der vierten Jg.; Jg. 4.2 = zweites Halbjahr der vierten Jg. 


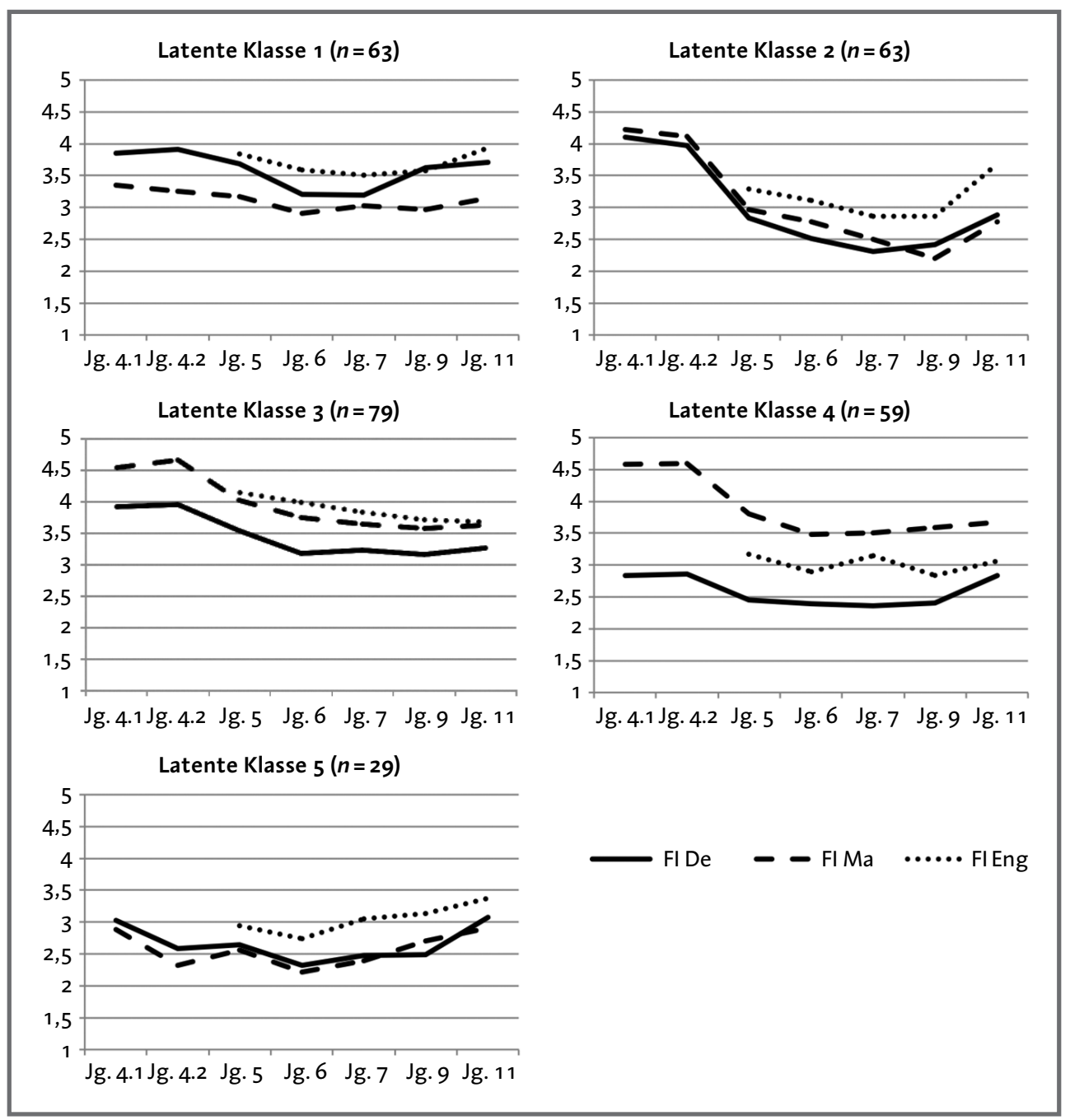

Abb. 1: Grafische Darstellung der mittleren Profilverläufe der Lösung mit fünf latenten Klassen.

Anmerkungen: Jg. = Jahrgangsstufe; Jg. 4.1 = erstes Halbjahr der vierten Jg.; Jg. 4.2 = zweites Halbjahr der vierten Jg.; FI De $=$ Fachinteresse Deutsch, FI Ma $=$ Fachinteresse Mathematik, FI Eng $=$ Fachinteresse Englisch.

zwei ( $n=63,54,0 \%$ weiblich) zeigen für die Fächerpaare Englisch und Mathematik sowie Englisch und Deutsch ein Profil der zunehmenden Differenzierung in Jahrgangsstufe 11. Der Verlauf der Fachinteressen Deutsch und Mathematik lässt sich hingegen als stabil undifferenziert bezeichnen. Beide Fachinteressen weisen in der Grundschule ein hohes Niveau auf, welches nach dem Übergang in die Sekundarstufe stark abfällt. Im Verlauf der Sekundar- stufe I unterliegen alle drei Fachinteressen einem (anhaltenden) negativen Entwicklungstrend. Erst im Übergang auf die Sekundarstufe II zeigt sich ein Wiederanstieg der mittleren Interessen, welcher für das Fachinteresse Englisch am stärksten ausfällt. Die bivariaten Korrelationen der Fachinteressen Englisch und Deutsch verweisen zwar deskriptiv ebenfalls auf einen abnehmenden Zusammenhang, dieser ist jedoch bei Betrachtung der Konfidenzintervalle 
Eine personenzentrierte Betrachtung der Entwicklung des Fachinteresses 11

Tab. 4: Bivariate Korrelationen der Fachinteressen Deutsch, Mathematik und Englisch innerhalb der latenten Klassen zu Jahrgangsstufe 4.1, 5 und 11

\begin{tabular}{|c|c|c|c|c|}
\hline Latente Klasse & Korrelation der Fachinteressen ... & Jg. 4.1 & Jg. 5 & Jg. 11 \\
\hline $\begin{array}{l}\text { Latente Klasse } 1 \\
(n=63)\end{array}$ & $\begin{array}{l}\text {...Deutsch und Mathematik } \\
\text {...Deutsch und Englisch } \\
\text {...Englisch und Mathematik }\end{array}$ & $\begin{array}{c}-.02 \\
- \\
-\end{array}$ & $\begin{array}{l}-.04 \\
-.12 \\
-.20\end{array}$ & $\begin{array}{r}.15 \\
.17 \\
-.02\end{array}$ \\
\hline $\begin{array}{l}\text { Latente Klasse } 2 \\
(n=63)\end{array}$ & $\begin{array}{l}\text {...Deutsch und Mathematik } \\
\text {...Deutsch und Englisch } \\
\text {...Englisch und Mathematik }\end{array}$ & $\begin{array}{c}-.16 \\
- \\
-\end{array}$ & $\begin{array}{l}.15 \\
.51^{*} \\
.11\end{array}$ & $\begin{array}{l}-.07 \\
.22^{+} \\
-.08\end{array}$ \\
\hline $\begin{array}{l}\text { Latente Klasse } 3 \\
(n=79)\end{array}$ & $\begin{array}{l}\text {...Deutsch und Mathematik } \\
\text {...Deutsch und Englisch } \\
\text {...Englisch und Mathematik }\end{array}$ & $\begin{array}{l}.15 \\
- \\
-\end{array}$ & $\begin{array}{l}.13 \\
.25^{*} \\
.01\end{array}$ & $\begin{array}{r}.11 \\
.07 \\
-.12\end{array}$ \\
\hline $\begin{array}{l}\text { Latente Klasse } 4 \\
(n=59)\end{array}$ & $\begin{array}{l}\text {...Deutsch und Mathematik } \\
\text {...Deutsch und Englisch } \\
\text {...Englisch und Mathematik }\end{array}$ & $\begin{array}{l}.26^{+} \\
- \\
-\end{array}$ & $\begin{array}{l}.27^{*} \\
.05 \\
.33^{*}\end{array}$ & $\begin{array}{r}-.02 \\
.13 \\
-.07\end{array}$ \\
\hline $\begin{array}{l}\text { Latente Klasse } 5 \\
(n=29)\end{array}$ & $\begin{array}{l}\text {...Deutsch und Mathematik } \\
\text {...Deutsch und Englisch } \\
\text {...Englisch und Mathematik }\end{array}$ & $\begin{array}{l}36^{+} \\
- \\
-\end{array}$ & $\begin{array}{l}.35^{+} \\
-.04 \\
-.16\end{array}$ & $\begin{array}{l}-.37^{+} \\
-.15 \\
-.31\end{array}$ \\
\hline
\end{tabular}

Anmerkungen: Jg. = Jahrgangsstufe; Jg. 4.1 = erstes Halbjahr der vierten Jg.; Jg. 4.2 = zweites Halbjahr der vierten Jg. ${ }^{+} p<.10 .{ }^{*} p<.05$.

nicht signifikant, Jahrgangsstufe 5: $r=.51(.30$; $.68), p<.05$; Jahrgangsstufe $11: r=.22(-.04 ; .45)$, $p>.05$. Die übrigen Fächerpaare korrelieren nicht signifikant. Das Profil der latenten Klasse drei ( $n=79,40,5 \%$ weiblich) kann mit Bezug auf die Mittelwertvergleiche der Fachinteressen Deutsch und Mathematik als zunehmend undifferenziert beschrieben werden. Beide Fachinteressen liegen zu Beginn des Erhebungszeitraums auf einem sehr hohen Niveau und sinken nach dem Übergang in die Sekundarstufe auf ein mittleres Niveau, welches dann bis zum Ende des Erhebungszeitraums stabil bleibt. Auch das Fachinteresse Englisch sinkt im Verlauf der Sekundarstufe von einem hohen Niveau leicht ab. Der Verlauf der Fachinteressen Mathematik und Englisch sowie Deutsch und Englisch kann als stabil undifferenziert beschrieben werden. Die Analyse der Korrelationen zeigt für die Fächerpaare Mathematik und Englisch sowie Deutsch und Mathematik keine signifikanten Korrelationen. Für das Fachinteresse Englisch und Deutsch zeigt sich ein signifikanter Zusammenhang in Jahrgangsstufe $5, r=.25$ (.02; .46), $p<.05$. Dieser sinkt in Jahrgangsstufe 11 auf einen Wert nahe null, $r=.07(-.15 ; .29), p>.05$.
Aufgrund der Überschneidung der Konfidenzintervalle der Korrelationswerte ist dieses deskriptive Muster einer zunehmenden Differenzierung nicht signifikant. Die Mittelwertbetrachtung der latenten Klasse vier $(n=59$, 23,7\% weiblich) ergibt für das Fachinteresse Deutsch und Mathematik bis Jahrgangsstufe 9 ein stabil differenziertes Profil. In Jahrgangsstufe 11 zeigt sich hingegen ein zunehmend undifferenziertes Entwicklungsmuster. Das Fachinteresse Mathematik verläuft hierbei in der Grundschule auf einem sehr hohen und in der Sekundarstufe auf einem mittleren Niveau. Die Entwicklung des Fachinteresses Deutsch findet hingegen auf einem niedrigen bis sehr niedrigen Niveau statt und zeigt erst in Jahrgangsstufe 11 einen Anstieg. Das Fachinteresse Englisch verläuft zwischen diesen beiden schulfachbezogenen Interessen auf einem mittleren Niveau und kann bezüglich beider Fachinteressen als stabil undifferenziert beschrieben werden. Die bivariaten Korrelationen der Fachinteressen Englisch und Mathematik verweisen auf einen mittleren Zusammenhang in Jahrgangsstufe 5, $r=.33(.07 ; .55), p<.05$, welcher in Jahrgangsstufe 11 auf einen Wert leicht unter null ab- 
sinkt, $r=-.07(-.33 ; .20), p>.05$. Wird aufgrund der geringen Fallzahl ein zehnprozentiges Signifikanzniveau berücksichtigt, zeigt sich dieses Muster auch für die Fachinteressen Deutsch und Mathematik, Jahrgangsstufe 4.1:r=.26 $(-.02 ; .50), p>.05 ;$ Jahrgangsstufe $11: r=-.02$ $(-.27 ; .24), p>.05$. Da sich jedoch die jeweiligen Konfidenzintervalle überschneiden, verweisen die Korrelationen beider Fächerpaare zwar deskriptiv, aber nicht signifikant auf eine zunehmende Differenzierung. Für das Fachinteresse Deutsch und Englisch zeigen sich keine signifikanten Korrelationen. Schließlich lässt sich das Entwicklungsprofil der Fachinteressen innerhalb der latenten Klasse $5(n=29$, $55,2 \%$ weiblich) mit Bezug auf die Mittelwertvergleiche als stabil undifferenziert beschreiben. Alle drei Fachinteressen verlaufen zudem auf einem niedrigen Niveau, wobei das Fachinteresse Englisch leicht positivere Werte als die Fachinteressen Deutsch und Mathematik aufweist. Die Betrachtung der Korrelationen zeigt keine signifikanten Zusammenhänge auf. Berücksichtigt man jedoch erneut ein zehnprozentiges Signifikanzniveau aufgrund der geringen Fallzahl, zeigt sich für das Fachinteresse Deutsch und Mathematik zum ersten Halbjahr der vierten Jahrgangsstufe ein mittlerer positiver $\mathrm{Zu}$ sammenhang, $r=.36(-.03 ; .65), p>.05$. Dieser kehrt sich in Jahrgangsstufe 11 in einen mittleren negativen Zusammenhang um, $r=-.37$ $(-.65 ; .01), p>.05$. Die Konfidenzintervalle beider Korrelationswerte überschneiden sich jedoch knapp, sodass das deskriptive Korrelationsmuster einer zunehmenden Differenzierung der Fachinteressen Deutsch und Mathematik nicht signifikant ist.

\section{Diskussion}

Ziel des vorliegenden Beitrages war es, den Entwicklungsverlauf der Fachinteressen Deutsch, Mathematik und Englisch von Jahrgangsstufe 4 bis 11 nachzuzeichnen. Hierbei sollte über die Betrachtung mittlerer Verläufe hinaus auch ein personenzentrierter Ansatz verfolgt werden. Bisherige Befunde der Interessensforschung be- stätigend, zeigten die Ergebnisse zunächst einen negativen Entwicklungstrend der mittleren Fachinteressen von der Grundschule bis in die Sekundarstufe I. Zudem konnte der leicht positive Entwicklungstrend der mittleren schulfachbezogenen Interessen nach dem Übergang in die Sekundarstufe II repliziert werden (Dotterer et al., 2009; Frenzel et al., 2010). Die Korrelationen zu den jeweils ersten und letzten Erhebungszeitpunkten innerhalb der Gesamtstichprobe verwiesen für die Fachinteressen Englisch und Mathematik auf einen abnehmenden Zusammenhang und deuten somit entsprechend bisheriger Studien auf eine zunehmende Interessensdifferenzierung im späten Schulverlauf hin (vgl. Baumert \& Köller, 1998). Der ebenfalls leicht abnehmende Zusammenhang der Fachinteressen Deutsch und Englisch erwies sich hingegen mit Blick auf die berechneten Konfidenzintervalle als nicht signifikant. Zwischen den Fachinteressen Deutsch und Mathematik gab es zu den jeweiligen Erhebungszeitpunkten keine bedeutsamen Zusammenhänge. Die Ergebnisse der anschließenden latenten Klassenanalyse mit längsschnittlichen Daten verwiesen auf relevante Subgruppen von Schülerinnen und Schülern mit unterschiedlichen Entwicklungsverläufen für die drei analysierten Fachinteressen. Anhand der statistischen Kennwerte sowie inhaltlich-theoretischer Gesichtspunkte wurde die Lösung mit fünf latenten Klassen ausgewählt. Die Charakterisierung der Entwicklungsprofile der latenten Klassen ergab mit Blick auf die Mittelwertvergleiche zwei latente Klassen, deren Profile zu Jahrgangsstufe 11 auf einen Prozess der zunehmenden Differenzierung verwiesen. Die latente Klasse 1 zeigte eine entsprechende Entwicklung für die Fachinteressen Englisch und Mathematik, die latente Klasse 2 zeigte ein Muster der zunehmenden Differenzierung für die Fachinteressen Englisch und Mathematik sowie Englisch und Deutsch. In allen drei Fällen lässt sich dieser Entwicklungsverlauf auf den vergleichsweise stärkeren Zuwachs des Fachinteresses Englisch im Gegensatz zu den jeweils anderen Fachinteressen zu Jahrgangsstufe 11 zurückführen. 
Deskriptiv zeigt sich dieses Befundmuster für die latente Klasse 2 auch bei der Analyse der bivariaten Korrelationen. Allerdings erwies sich die Veränderung der Korrelationswerte als nicht signifikant. Die latenten Klassen 3 und 4 zeigten für die Fachinteressen Mathematik und Deutsch ein Entwicklungsmuster, welches als zunehmend undifferenziert beschrieben werden kann. In beiden Fällen lässt sich dieser Befund auf den stärkeren Anstieg des Deutschinteresses im Gegensatz zum Mathematikinteresse in Jahrgangsstufe 11 zurückführen. Der Vergleich der Korrelationswerte verwies erneut auf keine signifikanten Veränderungen. Schließlich zeigte die latente Klasse 5 ein stabil undifferenziertes Interessensprofil auf einem niedrigen Niveau.

Das Ziel des vorliegenden Beitrags war es, durch ein personenzentriertes Analysedesign Subgruppen von Schülerinnen und Schülern mit spezifischen strukturellen Verläufen der Interessensentwicklung aufzeigen zu können. Hierbei sollte anhand von Mittelwertvergleichen sowie der ergänzenden Betrachtung bivariater Korrelationen der Frage nachgegangen werden, ob sich ein Muster der zunehmenden Differenzierung schulischer Interessen im (späteren) Schulverlauf aufzeigen lässt. Der Vergleich der Korrelationswerte ergab durchweg nicht signifikante Veränderungen, und auch die bivariaten Korrelationen zu den einzelnen Erhebungszeitpunkten waren mehrheitlich nicht signifikant. Beides lässt sich vermutlich auf die geringe Fallzahl innerhalb der einzelnen latenten Klassen zurückführen. Es ist jedoch generell an dieser Stelle festzuhalten, dass ein Prozess der Interessensdifferenzierung auch bei Unabhängigkeit der Fächerpaare stattfinden kann. Die Betrachtung der Korrelationen stellt zwar ein ergänzendes Maß für die Differenzierung dar, bei der eine absinkende Korrelation zwischen zwei Fachinteressen als Anzeichen für eine zunehmende Differenzierung im Zeitverlauf anzusehen ist (vgl. Baumert \& Köller, 1998; Daniels, 2008). Es kann jedoch nicht geschlussfolgert werden, dass ein anderes Korrelationsmuster einen Prozess der Interessens- differenzierung ausschließen würde. Vor diesem Hintergrund erscheint die Analyse der Mittelwertunterschiede innerhalb der einzelnen latenten Klassen ein geeignetes Instrument zu sein, um die Entwicklungsverläufe der analysierten Fachinteressen zu charakterisieren und eventuelle Differenzierungsprozesse nachzuzeichnen. Mit Bezug auf die Frage einer geschlechterstereotypen Interessensentwicklung ergaben sich zwei latente Klassen mit einem unausgeglichenen Geschlechterverhältnis. Innerhalb der latenten Klasse 4 befanden sich nur knapp ein Viertel Mädchen. Bisherige Befunde zur geschlechtsspezifischen Interessensentwicklung bestätigend verlief in dieser latenten Klasse das Fachinteresse Mathematik deutlich oberhalb des Fachinteresses Deutsch. Die latente Klasse 1 hat hingegen mit knapp $60 \%$ einen erhöhten Mädchenanteil und zeigt erwartungsgemäß erhöhte Interessenswerte für die verbalen Fächer Englisch und Deutsch (vgl. Kessels \& Hannover, 2004, 2006). Zudem verweisen die Profile beider latenter Klassen darauf, dass der Abfall der Fachinteressen in der vorrangig von Mädchen besetzten latenten Klasse deutlich flacher verlief als in derjenigen, welche vorrangig von Jungen besetzt war (vgl. Helmke, 1993). Darüber hinaus können die dargelegten Befunde einer zunehmenden Interessensdifferenzierung vor dem Hintergrund der Frage betrachtet werden, bei welcher Fächerkombination dieses Befundmuster vorrangig auftritt. Hierbei zeigte sich, dass dieses mehrheitlich zwischen Fächerpaaren der verbalen und mathematischen Domäne beobachtet werden konnte - ein Muster, welches von bisherigen Befunden aus der Selbstkonzeptforschung bestätigt wird (Möller \& Marsh, 2013).

Zusammenfassend lässt sich somit festhalten, dass bei Betrachtung der Mittelwertunterschiede zu den jeweils ersten und letzten Erhebungszeitpunkten die Befunde bei zwei latenten Klassen auf einen Prozess der zunehmenden Differenzierung zu Jahrgangsstufe 11 hindeuten. Die Analyse der Korrelationsmuster zu denselben Erhebungszeitpunkten ergab für die Fachinteressen Englisch und Mathematik in- 
nerhalb der Gesamtstichprobe ein signifikantes Muster der zunehmenden Differenzierung. Es kann somit festgehalten werden, dass zum Ende der Sekundarstufe zumindest für eine Subgruppe der Schülerinnen und Schüler tatsächlich noch einmal ein Prozess der Interessensdifferenzierung stattzufinden scheint. Dieser lässt sich anscheinend auf einen unterschiedlich stark akzentuierten Wiederanstieg der einzelnen Fachinteressen zurückführen. Es sei an dieser Stelle noch einmal darauf hingewiesen, dass keine der latenten Klassen einen negativen Entwicklungstrend der Fachinteressen zu Jahrgangsstufe 11 aufzeigte, sodass sich der mittlere Wiederanstieg der Fachinteressen nach dem Übergang in die Sekundarstufe II als sehr stabil erwies. Allerdings kann bei dem hier verwendeten Längsschnitt nicht ausgeschlossen werden, dass der beobachtete späte Wiederanstieg der Interessen auf einen selektiven Stichprobenausfall zurückzuführen ist. Dieser Erklärungsansatz konnte jedoch innerhalb der Studie von Dotterer und Kollegen (2009) explizit ausgeschlossen werden, sodass der Befund eines späten kurvenlinearen Verlaufs der Interessensentwicklung als bestätigt angesehen werden kann und somit weiterhin einer theoretischen Erklärung bedarf. Eventuell bietet der mit dem Übergang in die Sekundarstufe II verbundene Wechsel in der Unterrichtsorganisation einen Erklärungsansatz. Obwohl die hier analysierten Fächer in den Bundesländern Bayern und Hessen Pflichtfächer darstellen und somit nicht abgewählt werden können, beginnt mit der Sekundarstufe II das Kurssystem. Dieser Organisationswechsel und die damit verbundenen Neuerungen in bisher festgefahrenen Strukturen könnten mit einem generellen Motivationsanstieg für schulische Fächer verbunden sein.

Abschließend kann festgehalten werden, dass die hier aufgezeigten Befunde differenzieller Entwicklungsverläufe der Fachinteressen Deutsch, Mathematik und Englisch innerhalb von fünf latenten Klassen im Einklang mit denjenigen von Schurtz und Artelt (2014) stehen: Es zeigen sich - trotz der unterschied- lich verwendeten Stichprobengröße - vergleichbare Entwicklungsprofile für den jeweils gemeinsam analysierten Zeitraum von Jahrgangsstufe 4 bis 7. Die erweiterte Betrachtung der Interessensentwicklung von Jahrgangsstufe 7 bis 11 konnte darüber hinaus zeigen, dass sich die Interessen der Schülerinnen und Schüler innerhalb der fünf latenten Klassen in einer je spezifischen Weise weiterentwickeln. Demnach handelt es sich bei der hier erfolgten erweiterten Betrachtung der Interessensentwicklung um eine wichtige Fortführung der bereits von Schurtz und Artelt (2014) berichteten Entwicklungsprofile.

\section{Kritische Betrachtung der Befunde}

Einschränkend ist an dieser Stelle darauf hinzuweisen, dass das schulfachbezogene Interesse der Schülerinnen und Schüler auf einer aggregierten Ebene analysiert wurde. Obwohl diese Form der Operationalisierung bisherigen Studien zum mittleren Verlauf der Fachinteressen entspricht und somit eine Anschlussfähigkeit zu bereits publizierten Befunden gewährleistet (z. B. Daniels, 2008), vernachlässigt sie doch differenzielle Interessensbereiche innerhalb der jeweiligen Schulfächer. So verweisen bisherige Befunde darauf, dass die einzelnen Domänen eines Schulfaches mehr oder weniger interessant erscheinen können (Krapp, 2002). Für zukünftige Forschungsarbeiten wäre es demnach interessant zu analysieren, wie sich diese unterschiedlich stark akzentuierten Interessensbereiche zu einem Gesamturteil für das jeweilige Unterrichtsfach zusammenfügen. Zudem ist kritisch anzumerken, dass in der vorliegenden Arbeit lediglich drei schulfachbezogene Interessen analysiert wurden. Die gemeinsame Betrachtung der Entwicklung der Fachinteressen Deutsch, Mathematik und Englisch ermöglicht zwar einen ersten Einblick in differenzielle Prozesse der Interessensentwicklung, genügt jedoch nicht, um ein umfassendes Bild der schulfachbezogenen Interessensentwicklung nachzeichnen zu können. Schließlich sei an dieser Stelle noch einmal explizit darauf hinge- 
wiesen, dass die vorliegende Arbeit zwar die Befunde von Schurtz und Artelt (2014) bestätigt, es sich jedoch auch um Analysen derselben Längsschnittstudie handelt. Folglich steht noch eine Überprüfung der dargelegten differenziellen Entwicklungsprofile der Fachinteressen Deutsch, Mathematik und Englisch anhand einer unabhängigen Stichprobe aus. Hierbei sollte zudem berücksichtigt werden, dass die berichteten Befunde auf einer sehr kleinen Stichprobe von $n=293$ Fällen beruhen. Dies führte auch zu einer zum Teil sehr kleinen Besetzung der einzelnen latenten Klassen. Zudem konnte die LCA nur bis zu einer Anzahl von $\mathrm{k}=7$ latenten Klassen durchgeführt werden, was ebenfalls auf die geringe Stichprobengröße zurückgeführt werden könnte. Eine Replikation der dargelegten Analysen anhand einer größeren Stichprobe ist somit anzustreben. Trotz des geringen Stichprobenumfanges verweisen die Befunde von Nylund et al. (2007) darauf, dass dieser ausreichend für die Durchführung einer LCA ist. Darüber hinaus legen die Befunde von Kim (2012) dar, dass eine geringere Stichprobengröße erforderlich ist, wenn eine hohe Anzahl an Indikatoren vorliegt, die über einen möglichst langen Zeitraum gesammelt wurden. Da die analysierten Messzeitpunkte in dem vorliegenden Beitrag einen sechsjährigen Zeitraum umfassen, stützt dies die Angemessenheit der verwendeten Analysemethode zusätzlich.

\section{Zusammenfassung und Ausblick}

Trotz der dargelegten Einschränkungen liefern die Ergebnisse der vorliegenden Arbeit nicht nur eine wichtige Bestätigung bisheriger Befunde zu einem kurvenlinearen Verlauf der mittleren Interessensentwicklung im Schulverlauf, sondern verweisen darüber hinaus auf die Relevanz einer ergänzenden personenzentrierten Analyse der Interessensentwicklung. Letztere ermöglicht es, Subgruppen von Schülerinnen und Schülern mit differenziellen Entwicklungsverläufen auszumachen. Insbesondere vor dem Hintergrund, dass die Interessensentwicklung einen identitätsstiftenden und somit stark personalisierten Prozess darstellt, erscheint es notwendig, das vorherrschende Ungleichgewicht zwischen variablen- und personenzentrierten Ansätzen innerhalb der bisherigen Interessensforschung zugunsten Letzterer zu verschieben. Darüber hinaus konnte gezeigt werden, dass für die Betrachtung differenzieller Prozesse der Interessensentwicklung die Langzeitanalyse der Interessen im Schulverlauf notwendig ist. Insbesondere die Befunde der latenten Klassenanalyse mit längsschnittlichen Daten legen die Vermutung nahe, dass die Differenzierungsprozesse in einem ersten Schritt zu Beginn der Grundschule und in einem zweiten Schritt nach dem Übergang in die Sekundarstufe II stattfinden. Beide Zeitpunkte werden allerdings im vorliegenden Beitrag nur knapp beleuchtet, sodass eine fokussierte Betrachtung dieser beiden kritischen Zeitpunkte innerhalb zukünftiger Forschungsarbeiten zu wünschen ist.

\section{Literatur}

Alexandrowicz, R. (2008). Ein neuer Zugang zum BIC im Rahmen von Latent-Class-Analysen. In J. Reinecke \& C. Tarnai (Hrsg.), Klassifikationsanalysen in Theorie und Praxis (S. 141-165). Münster: Waxmann.

Archambault, I., Eccles, J.S. \& Vida, M. N. (2010). Ability self-concepts and subjective value in literacy: Join trajectories from grades 1 through 12. Journal of Educational Psychology, 102, 804-816. http://dx.doi.org/10. 1037/a0021075

Asparouhov, T. \& Muthén, B. (2012). Using Mplus TECH11 and TECH14 to test the number of latent classes (Mplus web notes, No. 14). Zugriff am 10.11. 2015 unter https://www.statmodel.com/examples/ webnotes/webnote14.pdf

Baumert, J., Gruehn, S., Heyn, S., Köller, O. \& Schnabel, K.-U. (1997). Bildungsverläufe und psychosoziale Entwicklung im Jugendalter (BIJU) Dokumentation: Band 1 , Skalen Längsschnitt I, Welle 1-4. Berlin: Max-PlankInstitut für Bildungsforschung.

Baumert, J. \& Köller, O. (1998). Interest research in secondary level I: An overview. In L. Hoffmann, A. Krapp, K. A. Renninger \& J. Baumert (Eds.), Interest and learning - Proceedings of the seeon conference on interest and gender (pp. 241-256). Kiel: Institute for Science Education at the University of Kiel.

Bühner, M. \& Ziegler, M. (2009). Statistik für Psychologen und Sozialwissenschaftler. München: Pearson Studium.

Daniels, Z. (2008). Entwicklung schulischer Interessen im Jugendalter. Münster: Waxmann.

Dickhäuser, O. (2003). Überprüfung des erweiterten Modells des internal/external frame of reference. Zeitschrift für Entwicklungspsychologie und Pädagogische Psycholo- 
gie, 35, 200-207. http://dx.doi.org/10.1026//00498637.35.4.200

Dotterer, A. M., McHale, S. M. \& Crouter, A.C. (2009). The development and correlates of academic interest from childhood through adolescence. Journal of Educational Psychology, 101, 509-519. http://dx.doi.org/ 10.1037/a0013987

Eccles, J.S., Midgley, C., Wigfield, A., Buchanan, C.M., Reuman, D., Flanagan, C. \& Mac Iver, D. (1993). Development during adolescence: The impact of stageenvironment fit on young adolescents' experiences in schools and in families. American Psychologist, 48 90-101. http://dx.doi.org/10.1037/0003-066X.48.2. 90

Frenzel, A.C., Goetz, T., Pekrun, R. \& Watt, H.M.G. (2010). Development of mathematics interest in adolescence: Influences of gender, family, and school context. Journal of Research on Adolescence, 20, 507 537. http://dx.doi.org/10.1111/j.1532-7795.2010.00 645.x

Frenzel, A. C., Pekrun, R., Dicke, A.-L. \& Goetz, T. (2012). Beyond quantitative decline: Conceptual shifts in adolescents' development of interest in mathematics. Developmental Psychology, 48, 1069-1082. http://dx. doi.org/10.1037/a0026895

Hartinger, A. \& Fölling-Albers, M. (2002). Schüler motivieren und interessieren - Ergebnisse aus der Forschung, Anregungen für die Praxis. Bad Heilbrunn: Julius Klinkhardt.

Helmke, A. (1993). Die Entwicklung der Lernfreude vom Kindergarten bis zur Klassenstufe 5. Zeitschrift für Pädagogische Psychologie, 7, 77-86.

Hoffmann, L. (2002). Promoting girls' interest and achievement in physics classes for beginners. Learning and Instruction, 12, 447-465. http://dx.doi.org/10.1016/ S0959-4752(01)00010-X

Jacobs, J.E., Lanza, S., Osgood, D. W., Eccles, J. S. \& Wigfield, A. (2002). Changes in children's self-competence and values: Gender and domain differences across grades one through twelve. Child Development, 73 509-527. http://dx.doi.org/10.1111/1467-8624.00 421

Kessels, U. \& Hannover, B. (2004). Entwicklung schulischer Interessen als Identitätsregulation. In J. Doll \& M. Prenzel (Hrsg.), Bildungsqualität von Schule: Lehrerprofessionalisierung, Unterrichtsentwicklung und Schulförderung als Strategien der Qualitätsverbesserung (S. 398-412). Münster: Waxmann.

Kessels, U. \& Hannover, B. (2006). Zum Einfluss des Image von mathematisch-naturwissenschaftlichen Schulfächern auf die schulische Interessenentwicklung. In M. Prenzel \& L. Allolio-Näcke (Hrsg.), Untersuchungen zur Bildungsqualität von Schule-Abschlussbericht des DFG-Schwerpunktprogramms (S. 352-369). Münster: Waxmann.

Kim, S.-Y. (2012). Sample size requirements in single- and multiphase growth mixture models: A Monte Carlo simulation study. Structural Equation Modeling: A Mul tidisciplinary Journal, 19, 457-476. http://dx.doi.org/ $10.1080 / 10705511.2012 .687672$

Krapp, A. (1992). Das Interessenkonstrukt: Bestimmungsmerkmale der Interessenhandlung und des individuellen Interesses aus der Sicht einer Person-Gegenstands-Konzeption. In A. Krapp \& M. Prenzel (Hrsg.), Interesse, Lernen, Leistung: Neuere Ansätze der pädagogisch-psychologischen Interessenforschung (S. 297-329). Münster: Aschendorff.
Krapp, A. (2002). Structural and dynamic aspects of interest development: Theoretical considerations from an ontogenetic perspective. Learning and Instruction, 12, 383-409. http://dx.doi.org/10.1016/S0959-4752 (01)00011-

Lo, Y., Mendell, N. \& Rubin, D. (2001). Testing the number of components in a normal mixture. Biometrika, 88, 767-778. http://dx.doi.org/10.1093/biomet/88. 3.767

Martin, D. P. \& Oertzen, T. von (2015). Growth mixture models outperform simpler clustering algorithms when detecting longitudinal heterogeneity, even with small sample sizes. Structural Equation Modeling: A Multidisciplinary Journal, 22, 264-275. http://dx.doi. org/10.1080/10705511.2014.936340

McLachlan, G. \& Peel, D. (2000). Finite mixture models. New York, NY: Wiley.

Meeus, W., Shoot, R. Van de, Klimstra, T. \& Branje, S. (2011). Personality types in adolescence: Change and stability and links with adjustment and relationships: A five-wave longitudinal study. Developmental Psycho$\operatorname{logy}, 47,1181-1195$. http://dx.doi.org/10.1037/a00 23816

Möller, J. \& Marsh, H. W. (2013). Dimensional comparison theory. Psychological Review, 120, 544-560. http:// dx.doi.org/10.1037/a0032459

Musu-Gillette, L.E., Wigfield, A., Harring, J. R. \& Eccles, J.S. (2015). Trajetories of change in students' selfconcepts of ability and values in math and college major choice. Educational Research and Evaluation, 21 , $343-370$.

Muthén, B. (2001). Latent variable mixture modeling. In G. Marcoulides \& R. Schumacker (Eds.), New developments and techniques in structural equation modeling (pp. 1-33). Mahwah, NJ: Erlbaum.

Muthén, B. (2007). Growth modeling of longitudinal data. Zugriff am 10.11.2015 unter http://www.statmodel. $\mathrm{com} /$ discussion/messages/14/2778.html?1423423694

Muthén, L. K. \& Muthén, B. (1998-2010). Mplus user's guide (6 ed.). Los Angeles, CA: Muthén \& Muthén.

Nylund, K. L., Asparouhov, T. \& Muthén, B. (2007). Deciding on the number of classes in latent class analysis and growth mixture modeling: A Monte Carlo simulation study. Structural Equation Modeling: A Multidisciplinary Journal, 14, 535-569. http://dx.doi.org/10. $1080 / 10705510701575396$

Peugh, J. \& Fan, X. (2012). How well does growth mixture modeling identify heterogeneous growth trajectories? A simulation study examining GMM's performance characteristics. Structural Equation Modeling: A Multidisciplinary Journal, 19, 204-226. http://dx.doi.org/ $10.1080 / 10705511.2012 .659618$

Pohlmann, B. (2005). Konsequenzen dimensionaler Vergleiche. Münster: Waxmann

Potvin, P. \& Hasni, A. (2014). Analysis of the decline in interest towards school science and technology from grades 5 through 11. Journal of Science Education and Technology, 23, 784-802. http://dx.doi.org/10.1007/ s10956-014-9512-x

Schurtz, I. M. \& Artelt, C. (2014). Die Entwicklung des Fachinteresses Deutsch, Mathematik und Englisch in der Adoleszenz: Ein personenzentrierter Ansatz. Diskurs Kindheits- und Jugendforschung, 9, 285-302.

Schurtz, I. M., Pfost, M., Nagengast, B. \& Artelt, C. (2014). Impact of social and dimensional comparisons on student's mathematical and English subject-interest at the beginning of secondary school. Learning and In- 
struction, 34, 32-41. https://doi.org/10.1016/j.learn instruc.2014.08.001

Todt, E. \& Schreiber, S. (1998). Development of interests. In L. Hoffmann, A. Krapp, K. A. Renninger \& J. Baumert (Eds.), Interest and learning: Proceedings of the Seeon conference on interest and gender (pp. 25-40). Kiel: Institute for Science Education at the University of Kiel.

Vuong, Q. (1989). Likelihood ratio tests for model selection and non-nested hypotheses. Econometrica, 57, 307-333.

Wang, J. \& Wang, X. (2012). Structural equation modeling: Applications using Mplus. Chichester: Wiley.
Dr. Irene M. Schiefer

Sarah Becker

Prof. Dr. Cordula Artelt

Lehrstuhl für Empirische Bildungsforschung

Otto-Friedrich-Universität Bamberg

Markusplatz 3

D-96045 Bamberg

E-Mail: irene.schiefer@uni-bamberg.de sarah.becker@uni-bamberg.de cordula.artelt@uni-bamberg.de 